\title{
Age-related DNA methylation changes are tissue-specific with ELOVL2 promoter methylation as exception
}

\author{
Roderick C. Slieker ${ }^{1 *} \mathbb{D}$, Caroline L. Relton² ${ }^{2}$ Tom R. Gaunt ${ }^{2}$, P. Eline Slagboom ${ }^{1}$ and Bastiaan T. Heijmans ${ }^{1}$
}

\begin{abstract}
Background: The well-established association of chronological age with changes in DNA methylation is primarily founded on the analysis of large sets of blood samples, while conclusions regarding tissue-specificity are typically based on small number of samples, tissues and CpGs. Here, we systematically investigate the tissue-specific character of age-related DNA methylation changes at the level of the $\mathrm{CpG}$, functional genomic region and nearest gene in a large dataset.

Results: We assembled a compendium of public data, encompassing genome-wide DNA methylation data (Illumina 450k array) on 8092 samples from 16 different tissues, including 7 tissues with moderate to high sample numbers (Dataset size range 96-1202, $N_{\text {total }}=2858$ ). In the 7 tissues (brain, buccal, liver, kidney, subcutaneous fat, monocytes and T-helper cells), we identified 7850 differentially methylated positions that gained (gain-aDMPs; cut-offs: $P_{\text {bonf }} \leq 0.05$, effect size $\geq 2 \% / 10$ years) and 4,287 that lost DNA methylation with age (loss-aDMPs), $92 \%$ of which had not previously been reported for whole blood. The majority of all aDMPs identified occurred in one tissue only (gainaDMPs: 85.2\%; loss-aDMPs: 97.4\%), an effect independent of statistical power. This striking tissue-specificity extended to both the functional genomic regions (defined by chromatin state segmentation) and the nearest gene. However, aDMPs did accumulate in regions with the same functional annotation across tissues, namely polycomb-repressed $\mathrm{CpG}$ islands for gain-aDMPs and regions marked by active histone modifications for loss-aDMPs.
\end{abstract}

Conclusion: Our analysis shows that age-related DNA methylation changes are highly tissue-specific. These results may guide the development of improved tissue-specific markers of chronological and, perhaps, biological age.

Keywords: DNA methylation, 450 k, Tissue-specific, Ageing

\section{Background}

The association between DNA methylation and age in humans is well established for whole blood [1-10], and also in adipose tissue, brain and mesenchymal stem cells, loci have been found where DNA methylation changes with age [11-13]. A prime example is the CpGs near the ELOVL2 gene that exhibit consistent age-related changes in blood, hMSCs [13] and teeth [14] and other tissues $[15,16]$, an association that even extends to tissue from

\footnotetext{
*Correspondence: r.c.slieker@lumc.n

${ }^{1}$ Molecular Epidemiology, Department of Biomedical Data Sciences, Leiden University Medical Center, Einthovenweg 20, 2333 ZC Leiden, The Netherlands

Full list of author information is available at the end of the article
}

another species, namely the mouse [17]. The strength of the associations has led to the development of multiple predictors that can accurately estimate chronological age from methylation levels at a limited set of $\mathrm{CpG}$ sites [18-20]. While most predictors are trained on wholeblood DNA methylation data, one age predictor works independent of tissue type [18-20]. Intuitively, the high precision of the tissue-independent age predictor may rely on combining the cumulative information of CpGs whose DNA methylation level changes with age in multiple tissues simultaneously [14]. However, current views of the extent of tissue-specificity versus tissue-shared age-related DNA methylation are based on relatively small-scale studies with repect to the number of samples,

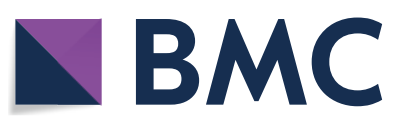

(c) The Author(s) 2018. This article is distributed under the terms of the Creative Commons Attribution 4.0 International License (http://creativecommons.org/licenses/by/4.0/), which permits unrestricted use, distribution, and reproduction in any medium, provided you give appropriate credit to the original author(s) and the source, provide a link to the Creative Commons license, and indicate if changes were made. The Creative Commons Public Domain Dedication waiver (http://creativecommons.org/ publicdomain/zero/1.0/) applies to the data made available in this article, unless otherwise stated. 
Table 1 Overview of studies of age-related DNA methylation changes in multiple tissues

\begin{tabular}{|c|c|c|c|c|c|c|}
\hline \multirow[t]{2}{*}{ Species } & \multirow[t]{2}{*}{ Tissues $(n)$} & \multirow[t]{2}{*}{ CpGs (Platform) } & \multicolumn{3}{|c|}{ Comparison of overlap at each level } & \multirow[t]{2}{*}{ Ref } \\
\hline & & & CpG & $\begin{array}{l}\text { Functional } \\
\text { genomic } \\
\text { region }\end{array}$ & Gene & \\
\hline \multicolumn{7}{|l|}{ Humans } \\
\hline Human & $\begin{array}{l}\text { Buccal (96), liver (147), kidney (171) } \\
\text { Th cells (214), brain (603), SC fat (648), monocytes (1202) }\end{array}$ & $\begin{array}{l}428,279 \\
\text { (Illumina } 450 \mathrm{k})\end{array}$ & + & + & + & Current study \\
\hline Human & $\begin{array}{l}\text { Cervix (3), bladder (5), intestine (5), kidney (6), head/neck } \\
\text { (11), brain (12), pleura (18), placenta (19), lung (49), blood } \\
\text { (85) }\end{array}$ & $\begin{array}{l}1413 \\
\text { (GoldenGate) }\end{array}$ & - & - & - & [21] \\
\hline Human & Muscle (51), blood (71), brain (78), kidney (83) & $\begin{array}{l}26,486 \\
(27 \text { k array })\end{array}$ & + & - & - (GO terms) & [22] \\
\hline Human & Neuron (29), glia (29), MSCs (92), whole blood (656) & $\begin{array}{l}429,789 \\
\text { (Illumina } 450 \mathrm{k})\end{array}$ & + & - & - & [13] \\
\hline \multicolumn{7}{|l|}{ Rodents } \\
\hline Rat & Fat (3), liver (5-6) & $\begin{array}{l}40,000 \\
\text { (HELP assay) }\end{array}$ & - & - & - & {$[45]$} \\
\hline Mouse & Liver (15), heart (15), lung (16), cortex (16) & $\begin{array}{l}1,230,000 \\
\text { (RRBS) }\end{array}$ & + & - & - (GO terms) & {$[46]$} \\
\hline
\end{tabular}

tissues and/or CpG sites (Table 1). The three previous human studies on tissue-specificity included between 4 and 92 samples per tissue (and 656 whole-blood samples) interrogating 1413, 26,486 and 429,789 CpG sites [13, 21, $22]$. Although two of these studies concluded that agerelated DNA methylation are tissue-specific [13, 21], the third reported that age-related changes were both shared across tissues and tissue-specific [22]. However, small numbers of tissues, samples and CpGs are biased towards finding tissue-specificity. Hence, conclusive evidence whether age-related changes are tissue-specific or tissueshared is lacking.

Here, we report on a systematic genome-wide analysis of age-related DNA methylation changes in a collection of 2858 methylomes from 7 tissues and show that the DNA methylation changes are highly tissue-specific and cannot be attributed to differences in statistical power. This tissue-specificity is not restricted to the individual CpG site but extends to the level of the functional region and the nearest gene to which a CpG maps. However, in every tissue, it is the same functional region (non-CGI, CGI, polycomb binding site, etc.) that accumulates agerelated changes albeit at distinct locations in the genome.

\section{Results}

To investigate age-related DNA methylation changes between tissues, Illumina 450k DNA methylation data were obtained from public repositories for 16 tissues encompassing in total 8092 individuals (Additional file 1: Table S1). First, we revisited the age-related differentially methylated position (aDMP) near ELOVL2 (cg16867657), to test whether the tissue-independent character of this
aDMP extended to multiple tissues. Gain of methylation was observed in blood ( $N=3295$, Fig. 1a) and extended to all tissues investigated except cerebellum (Fig. 1b) in line with previous reports [15].

To systematically study the occurrence of tissue-specific and tissue-shared aDMPs, we identified aDMPs in tissues for which a moderate to large sample size was available $(96 \leq N \leq 1202$; Additional file 1: Table S1), which included brain $(N=380)$, buccal $(N=96)$, liver $(N=147)$, kidney $(N=171)$, subcutaneous fat (SAT, $N=648)$, monocytes $(N=1202)$ and T-helper cells (Th cells, $N=214)$. As a comparison, whole-blood aDMPs were obtained from our previous work $(N=3295$, [23]). We focused on a conservative set of aDMPs defined by genome-wide significance $\left(P_{\text {bonf }} \leq 0.05\right)$ and a robust agerelated gain or loss that was larger than $2 \%$ per 10 years. Out of the 428,279 CpGs investigated, 7850 unique CpGs gained DNA methylation in one or more tissues (gainaDMPs) and 4287 unique CpGs lost DNA methylation in one or more tissues (loss-aDMPs). The number of aDMPs identified in each tissue varied strongly, with the highest number in buccal (4857 aDMPs in $N=96$; Fig. 2a, Additional File 2: Table S2) and the lowest number in Th cells (39 aDMPs in $N=214$, Additional File 2: Table S2). As expected, whole-blood showed a substantial overlap with monocytes (62 gain-aDMPs, 84 lossaDMPs, Additional File 2: Table S2) and Th cells (20 gain-aDMPs, 3 loss-aDMPs, Additional File 2: Table S2). Therefore, whole blood was not included in subsequent comparative analyses. The number of gain- versus lossDMPs differed between tissues. For example, in liver, aDMPs mainly gained DNA methylation (gain 2499, loss 

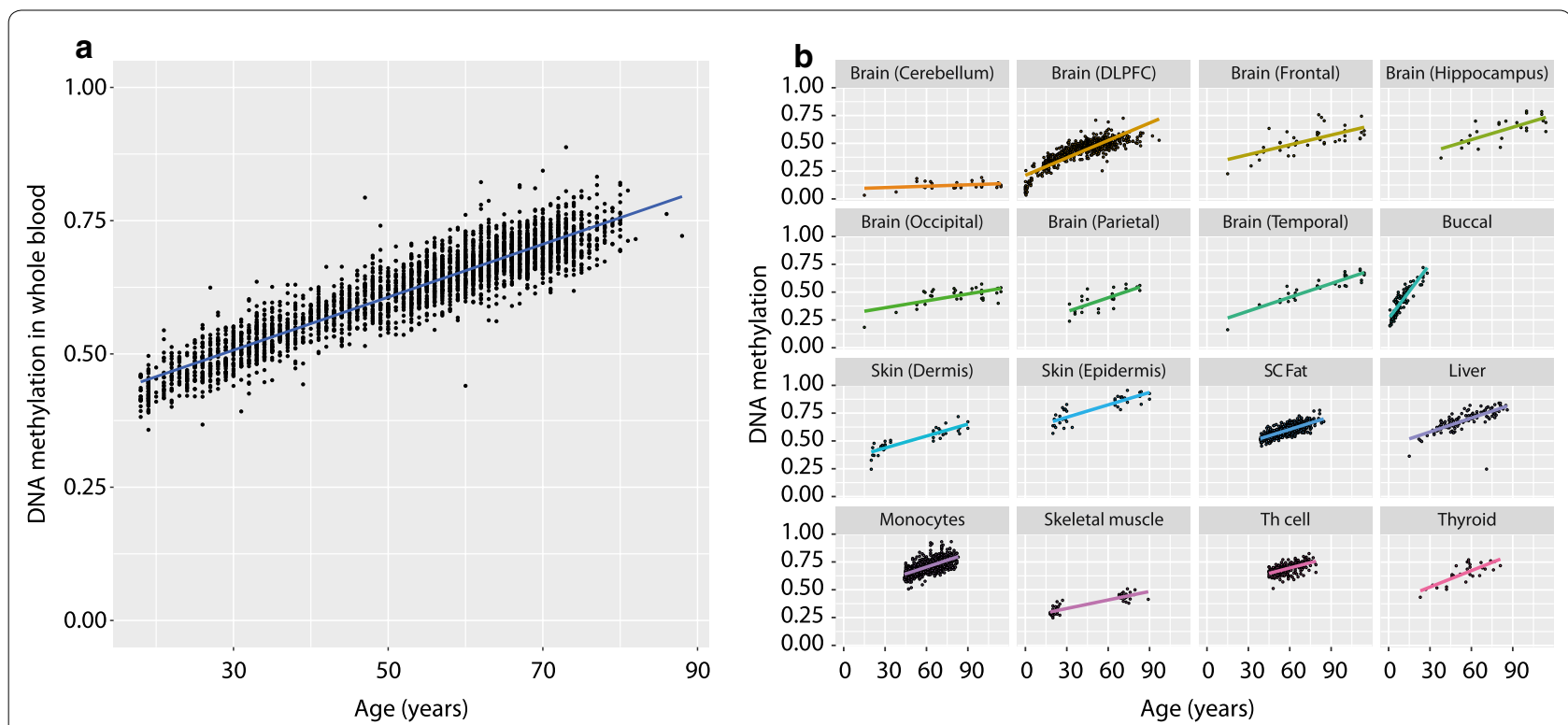

Fig. 1 Age-related change in DNA methylation in ELOVL2. a DNA methylation (y axis) against age ( $x$ axis) in blood for the ELOVL2 CpG (cg16867657). b DNA methylation (y axis) against age ( $x$ axis) in other tissues for the ELOVL2 CpG (cg16867657)
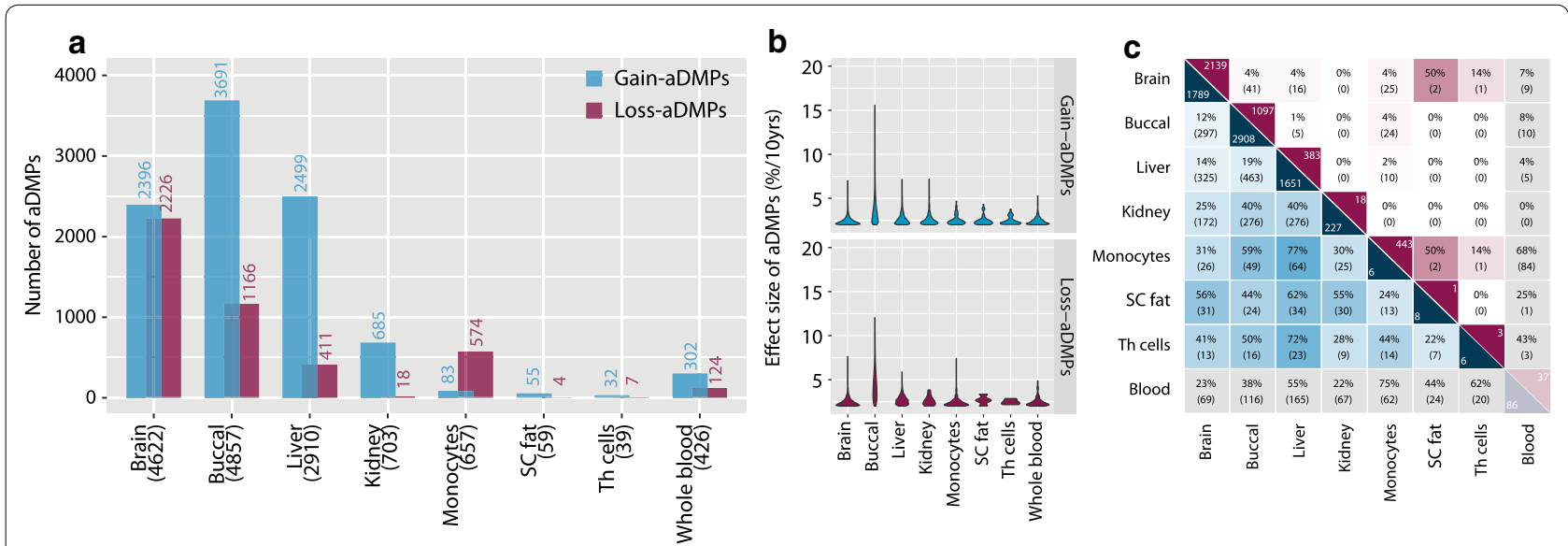

Fig. 2 Identification of aDMPs. a Number of identified gain- and loss-aDMPs ( $y$ axis) in this study for each tissue ( $x$ axis). b Slopes of identified gain- and loss-aDMPs (y axis) for each tissue ( $x$ axis). c Overlap between tissues in identified gain- and loss-aDMPs. In the diagonal cells the number of aDMPs unique for that tissue, the upper number represents the percentage, the lower number the number of overlapping aDMPs. Bluegain-aDMPs; Purple—loss-aDMPs

411; Fig. 2a, Additional File 2: Table S2), while in monocytes aDMPs mainly lost DNA methylation (gain 83, loss 574, Additional File 2: Table S2). Not only the number of DMPs but also the rates of change with age varied between tissues (Fig. 2b). The differences in number per tissue was not explained by either the known replication rate of the stem cells of the tissues analyzed $(r=-0.05$, $P=0.90$; Additional file 3: Fig. S1A) or the number of individuals used in each tissue $(r=-0.47, P=0.24$; Additional file 3: Fig. S1B).

\section{The majority of aDMPs are tissue-specific}

The comparison of aDMPs between tissues showed that the large majority of aDMPs were tissue-specific $(85.2 \%$ for gain-aDMPs and $97.4 \%$ for loss-aDMPs). Albeit low, the number of aDMPs shared between multiple tissues was higher for gain-aDMPs than for loss-aDMPs (Fig. 2c). Of the gain-aDMPs, $1161(14.8 \%)$ were identified in $\geq 2$ tissues (Fig. 3a). Only 2 gain-aDMPs were found in all 7 tissues studied and both mapped to the ELOVL2 locus (Additional file 4: Table S3), thus underscoring that the strong 


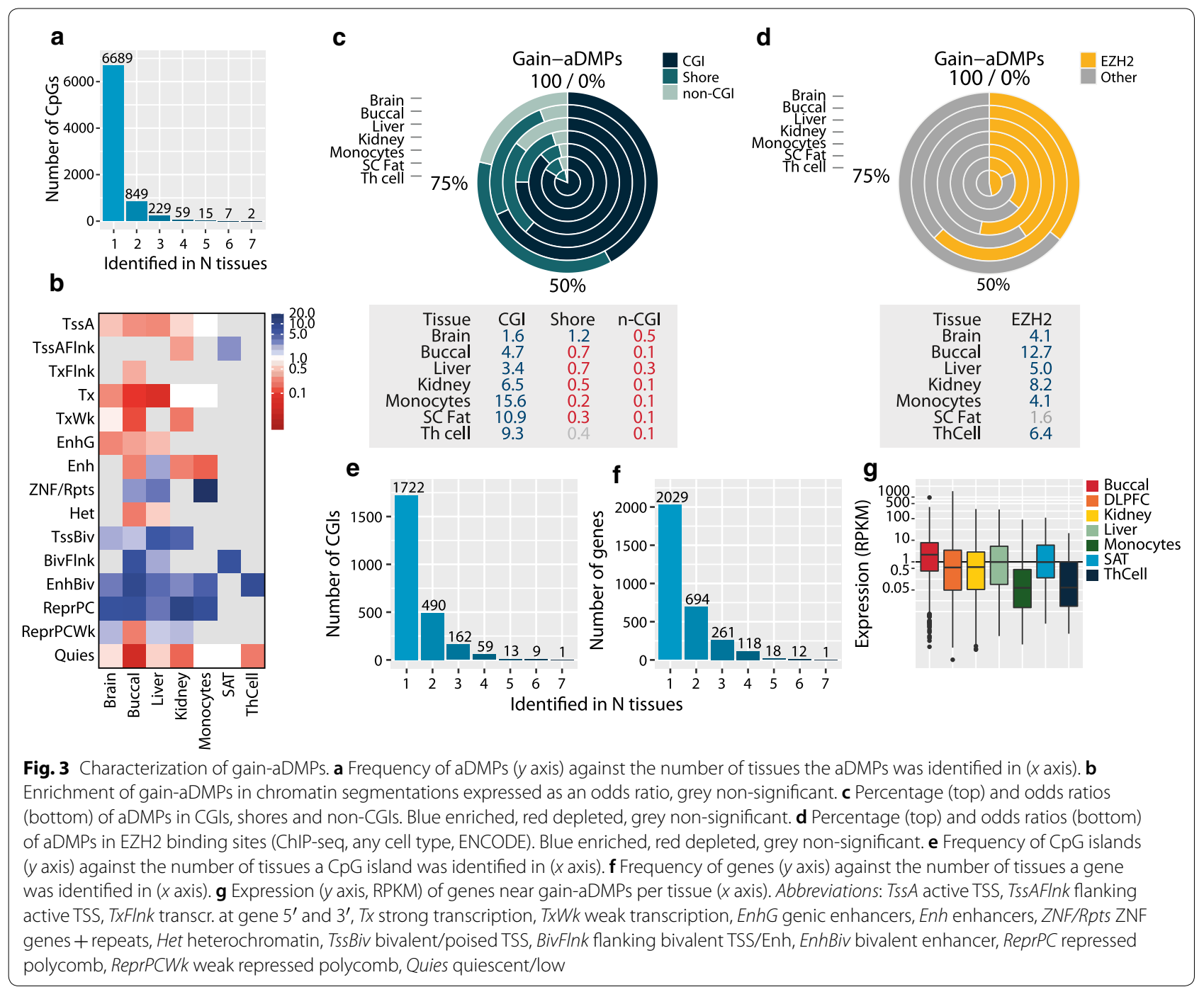

tissue-shared association of these CpGs with age is exceptional. Loci consistently identified in blood [3] were also found in a subset of the tissues, that is 971 aDMPs (8.0\%) overlapped with the 7477 CpGs previously identified in blood including FHL2 (5 tissues) and PENK (4 tissues).

To exclude that the tissue-specificity was only due to the differences in size of the datasets, we performed three additional analyses. First, we identified aDMPs based on an effect size criterion only, thus eliminating the effect of statistical power. While CpGs in each of the tissues have an equal chance to become an aDMP, tissue-specificity was again observed in each of the tissues in the 37,136 aDMPs identified (brain 4786, buccal 27,884, liver 4225, kidney 3694, monocytes 769 , SAT 222, Th cells 721; Additional file 5: Fig. S2A). Secondly, we identified aDMPs in equally sized datasets $(N=96$, based on smallest tissue datasets) with both a $P$ value $\left(P_{\text {bonf }} \leq 0.05\right)$ and effect size cut-off $(>2 \% / 10$ years $)$ criterion. This approach resulted in 10,249 aDMPs across the 7 tissues (brain 4195, buccal 4857, liver 1636, kidney 499, monocytes 109, SAT 23), and these aDMPs were equally tissue-specific between tissues (Additional file 5: Fig. S2B). In these equally sized datasets, we observed that the aDMPs identified in one tissue were significant in other tissues at a less stringent $P$ value cut-off $(P<0.001)$, suggesting that there may be a weak aDMP effect. However, this effect was much weaker as compared to the tissue the aDMP was identified in (Additional file 5: Fig. S2A-B, Additional file 6: Fig. S3).

Thirdly, the tissue-specificity of aDMPs was confirmed when we determined the age-related slope of the set of originally identified aDMPs in the all available 16 tissues (Additional file 6: Fig. S3). Together, these analyses reinforced the interpretation that aDMPs are truly tissue-specific and not due to differences in statistical power to detect aDMPs between tissues. Hence, age-related methylation 
changes occurring in one tissue are generally not indicative of age-related changes at the same CpGs in another tissue.

Identified aDMPs showed little overlap with the 353 CpGs from Horvath's age predictor [24] (Additional file 7: Fig. S4). The maximum overlap with gain-aDMPs was found in brain (13 gain-aDMPs) and with loss-aDMPs in monocytes (6 loss-aDMPs). This is not unexpected, given that Horvath's age predictor was trained using a penalized regression method aimed at identifying a sparse set of independent predictors and the fact that it was based on the Illumina $27 \mathrm{k}$ array (and hence did for example not include the ELOVL2 CpGs). When Horvath's tissueindependent age predictor was applied to the compendium of 16 tissues, the correlation between chronological age and predicted age was high although the precision of the prediction for individual samples was often limited (Additional file 8: Fig. S5). Only a minority of the CpGs that were included in Horvath's age predictor show a strong association with age and the strongest tissue-specific aDMPs are missing (Additional file 9: Fig. S6). This illustrates the potential value of tissue-specific analyses to gain insight into the mechanisms linking age-related DNA methylation changes with tissue-specific ageing.

\section{Gain-aDMPs are tissue-specific but share their functional annotation}

Genomic annotation showed that gain-aDMPs were highly enriched at CpG islands (CGIs) and their shores as compared with non-CGI sequences in each of the seven tissues (OR 1.6-15.6, $P<0.0001$, Fig. 3c) and also in whole blood (OR 17.5, $P<0.0001$, Additional file 10: Fig. S8A). These findings are in line with previous findings $[1,16,25]$. Utilizing reference chromatin segmentation data (marking the biological function of genomic regions) of primary tissues matching the tissues studied here [26], we found that gain-aDMPs preferentially occur at Bivalent Enhancers (OR 2.8-8.0, $P<0.0001$, Fig. 3b) and Repressed Polycomb (3.4-9.8, $P<0.0001$ ), both characterized by the polycomb repression mark H3K27me3. The latter observation was confirmed by the frequent co-occurrence of gain-aDMPs with binding sites of polycomb (PcG) repressive complex 2 (PRC2) protein EZH2. At least one-third of the identified gain-aDMPs overlapped with an EZH2 binding site increasing to almost two-thirds for buccal cells (OR 12.7, $P<0.0001$, Fig. 3d). To address whether the enrichments for CGIs and EZH2 binding are independent or reflect the same underlying biology, we analysed both annotations together. Gain-aDMPs were twofold enriched $(1.9-2.8, P<0.0001)$ at genomic regions that were both CGIs and binding EZH2 as compared with regions that featured only one of the annotations (Additional file 11: Fig. S7A). This suggests that gain-aDMPs primarily occur at regions are both polycomb-repressed and CGIs. Finally, when the same enrichment analyses were performed on the whole-blood aDMPs, similar results were observed as compared to the other seven tissues (Additional file 10: Fig. S8A-C).

\section{CGIs and genes that gain methylation are also tissue-specific}

Our analysis showed that, although individual gainaDMPs are tissue-specific, their genomic annotation is shared. This was not due to different CpGs in the same CGI being identified as aDMPs across different tissues. This would go against the interpretation that gain-aDMPs are mainly tissue-specific. However, this was not the case. Of the 1,722 CGIs harbouring at least one gain-aDMP in at least one tissue, $70.1 \%$ were unique (Fig. 3e). The tissue-specificity further extended towards genes: mapping gain-aDMPs to their nearest gene, resulting in 2029 genes that were unique for a tissue $(64.8 \%)$. Only one gene was found in all 7 tissues, namely (as expected) the ELOVL2 gene (Fig. 3f and Additional file 4: Table S3). The 12 genes that were identified in 6 out of 7 tissues included BMII (involved in the DNA damage response) and LIN28B (a microRNA that enhances IGF-2 translation). Counting the number of gain-aDMPs near a gene per tissue corroborated the tissue-specificity of genes. Genes with $>5$ gainaDMPs in one tissue had few in other tissues (Additional file 12: Table S4). Examples were PRRT1 in the brain (brain 24 , buccal 5 , liver 7 , kidney 1 , monocytes 0 , SAT 1 , Th cell 2) and HOXD in buccal cells (buccal 26, other tissues 0 ).

Next, we investigated the function of genes near gainaDMPs. In brain (84 processes), buccal (151 processes), liver (64 processes) and kidney (59 processes), multiple biological processes were enriched among nearest genes $\left(P_{\text {bonf }} \leq 0.05\right)$. Commonly and strongly enriched processes included embryonic morphogenesis (number of genes in brain 82 , buccal 98, liver 69, kidney 37; $P_{\text {bonf }}<0.0001$, Additional file 13: Table S5) and regulation of transcription (number of genes in brain 231, buccal 318, liver 209, kidney 134; $P_{\text {bonf }}<0.0001$, Additional file 13: Table S5).

Finally, we investigated the expression of genes near gain-aDMPs using public gene expression data on tissues matching those studied here (GTEX data, frontal cortex, $N=108$; oesophagus-mucosa, $N=286$; liver, $N=119$; kidney cortex, $N=32$; whole blood, $N=393$; age range 20-79 years). The baseline expression of genes was low (in line with their repressed state and developmental function), and we did not observe evidence for changes in gene expression (Fig. 3g and Additional file 11: Fig. S7B). Furthermore, there was little overlap between these genes and those previously reported to have a changed expression with age in whole blood (brain 91, buccal 104, liver 88 , kidney 28 , monocyte 1 , SAT 3 , Th cell 0) [27]. 
Loss-aDMPs are enriched for active regions including tissue-specific enhancers

In contrast to gain-aDMPs, loss-aDMPs preferentially occurred in non-CGI regions (OR 1.3-9.1, $P<0.0001$, Fig. 4a) in the seven tissues and in whole blood (Additional file 10: Fig. S8D), in line with previous reports [1, $16,25]$. Loss-aDMPs were even more tissue-specific than gain-aDMPs: 4,176 loss-aDMPs (97.4\%) were unique for one tissue and only 111 loss-aDMPs (2.6\%) were found in $\geq 2$ tissues (Fig. 4b). Again, contrasting with gainaDMPs, loss-aDMPs were particularly overrepresented at chromatin states marking active genomic regions (Fig. 4c). In 5 of 7 tissues, an enrichment was found for Enhancers including in brain (OR 6.6, $P<0.0001$, Fig. 4c), buccal cells (OR 2.7, $P<0.0001$ ), liver (OR 1.6, $P<0.001$ ), monocytes (OR 2.9, $P<0.0001)$ and Th cells (OR 11.2, $P<0.001)$. For 2 tissues, an enrichment for Genic enhancers was observed including brain (OR 11.9, $P<0.0001$ )

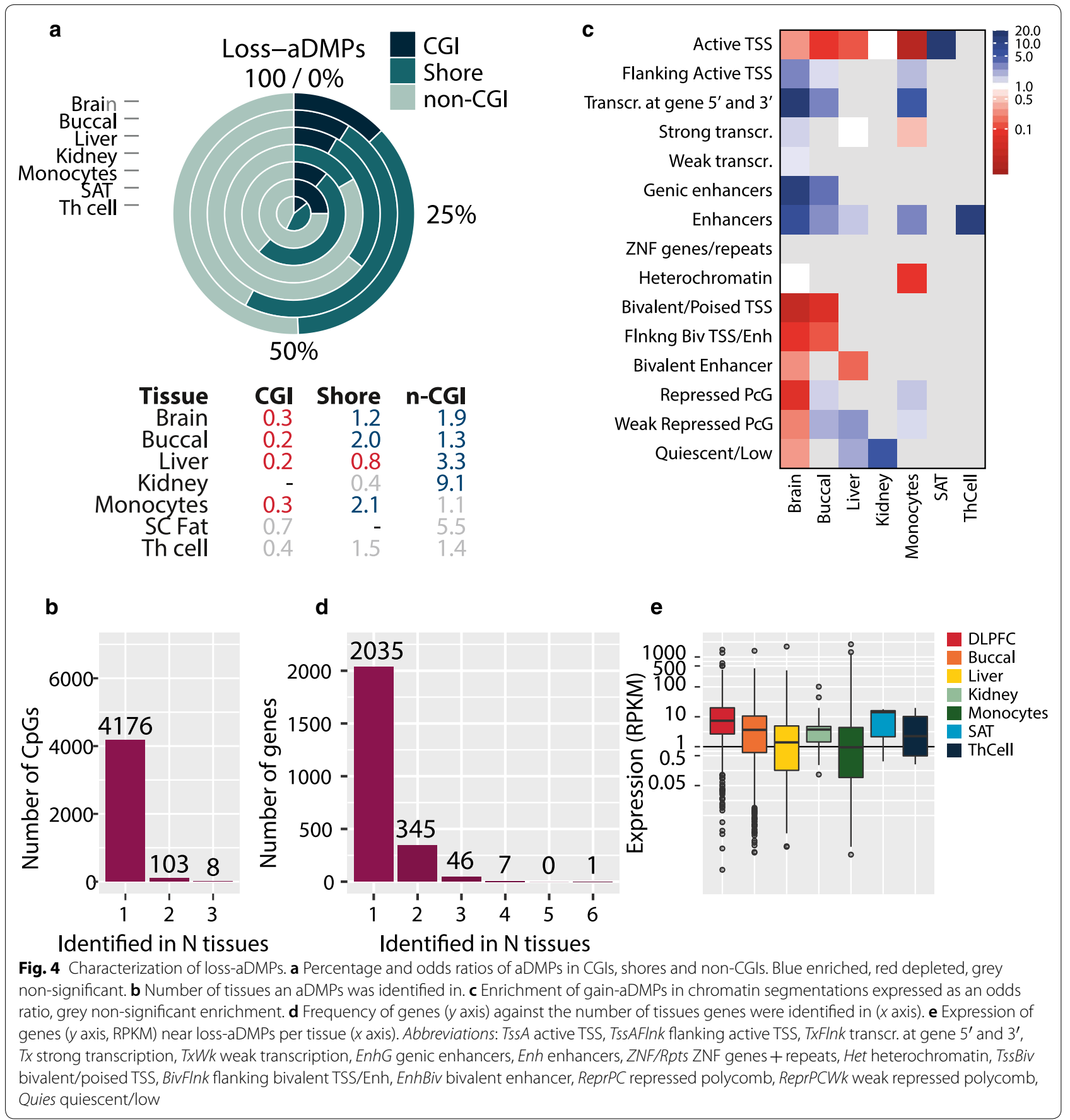


and buccal (OR 3.6, $P<0.0001$ ). Moreover, loss-aDMPs were overrepresented at actively transcribed regions, including Transcribed at $3^{\prime}$ and $5^{\prime}$ in brain (OR 14.5, $P<0.0001$ ), buccal (OR 3.0, $P<0.0001$ ) and monocytes (OR 4.6, $P<0.01$ ). Again, these observations were comparable with enrichments for aDMPs in whole blood (Additional file 10: Fig. S8E).

Mapping loss-aDMPs to their nearest gene showed that the majority of genes uniquely occurred in a single tissue (2035 genes, 83.6\%, Fig. 4d). The relatively few genes found in multiple tissues included $C D 46$ observed in 6 out of 7 tissues and KCNQ1, FAM92B, PLEC, GSE1, BAIAP2, PRDM16 and ACTG1 found in 4 tissues (Additional file 14: Table S6). Many of these genes have a 'housekeeping' function. For example, PLEC, BAIAP2, $A C T G 1$ play a role in the maintenance of the cytoskeleton. The tissue-specificity of loss-aDMP genes was corroborated when counting the number of loss-aDMPs per gene. For example, 24 loss-aDMPs were identified near $D I P 2 C$ in the brain, against low numbers in other tissues (buccal 1 , liver 3 , kidney 0 , monocytes 0 , SAT 0 , Th cell 0). In buccal, $18 \mathrm{CpGs}$ lost DNA methylation near SLC7A5, while no loss-aDMPs were found near this gene in other tissues (Additional file 15: Table S7).

Only loss-aDMP genes in brain showed enrichment for specific biological processes, including regulation of Small GTPase-mediated signal transduction (43 genes, $P_{\text {bonf }}<0.0001$ ) and Regulation of cell motion (33 genes, $P_{\text {bonf }}<0.05$, Additional file 16: Table S8). For the other tissues, similar processes related to intracellular signalling and cell motility were overrepresented $(P<0.05)$.

Finally, we investigated the expression of genes near loss-aDMPs. Their expression levels were moderate (Fig. 4e), but no evidence was observed for age-related changes (Additional file 11: Fig. S7C). Also, only a limited overlap was found with previously identified age-related differentially expressed genes in whole blood (brain 114; buccal 77 , liver 23 , kidney 3 , monocyte 36 , SAT 0 , Th cell 1) [27].

\section{Discussion}

Using genome-wide DNA methylation data on a large number of individuals and 16 tissues, we report a catalogue of 7850 robust aDMPs, $92 \%$ of which had not been previously reported in studies of whole blood, and show that age-related changes in DNA methylation are highly tissue-specific. The exceptions to this are well-known CpGs in the ELOVL2 promoter that display an exceptionally consistent increase in DNA methylation with age in all tissues studied here [15]. Age-related gain of DNA methylation (gain-aDMPs) accumulated at CpG islands and their flanking regions that were bound by the repressive PRC2 component EZH2. In contrast, loss-aDMPs were enriched for active regions, including enhancers. Underscoring the tissue-specificity of aDMPs, we observed that the large majority of both CGIs and genes with at least one aDMP were observed in a single tissue only.

Our results raise the question of what mechanism drives the age-related DNA methylation changes. Despite the tissue-specificity of individual aDMPs, in all tissues it was the same type of functional genomic region that accumulated aDMPs. We were able to exclude differences in the number of stem cell divisions between tissues as a potential explanation. In line with our findings for gain-aDMPs, age-related changes at regions marked by polycomb have been found in many studies investigating blood $[7,9,10,28]$ and have been found in other species $[17,29]$. A previously proposed explanation for the gain of DNA methylation in CpG islands is by loss of binding - or erosion - of the polycomb repressive complex 2 protein from the DNA (PRC2) [30]. CGI promoters of developmental genes that are expressed at low levels are kept in a repressive state primarily by the repressive complex PRC2 of which EHZ2 is a key component. Age-related loss of repression would allow DNA methyltransferases (DNMTs) to de novo methylate CGIs [30]. This explanation, however, requires a region and tissue-specific mechanism that renders a subset of regions more susceptible to age-related erosion of PRC2, which is currently unknown. While the genomic annotations that show age-related gain across tissues were the same, the actual loci were tissue-specific, suggesting common underlying mechanisms that link to tissue-specific agerelated changes.

In contrast to gain-aDMPs, loss-aDMPs overlapped with active genomic regions, such as enhancers, corroborating earlier studies in whole blood and mesenchymal stem cells [10, 13, 27]. Despite their tissue-specificity, genes near loss-aDMPs were not enriched for features conveying a role in tissue-specific processes, but instead with generic processes such as intracellular signalling cascade and cell motility pathways in line with findings in whole blood [3].

Remarkably, we did not find evidence for age-related changes in expression of genes near aDMPs. This confirms previous studies that aDMPs, including ELOVL2, have limited functional consequences [3, 9]. An explanation for the consistent increase in DMPs near ELOVL2 and other age-related DNA methylation changes could be due to underlying mitotic changes, although this has not been observed in previous studies suggesting other mechanisms driving age-related DNA methylation changes [31, 32]. In contrast to aDMPs, CpGs accumulating variability in the population with age (aVMPs) are commonly associated with gene expression changes and may be more 
informative for biological age [23]. Nonetheless, agerelated epigenetic changes show resemblance with the changes seen in cancer and cellular senescence [28]. For example, the number of passages in vitro can be tracked based on the changes that occur at the DNA methylation level [33]. Moreover, cellular senescence is associated with hypermethylation of CGIs and flanking regions, while hypomethylation occurs at non-CGI features [34, 35]. Cancer is also characterized by hypermethylation of $\mathrm{CpG}$ islands and global hypomethylation [36, 37]. Here, we observed a higher fraction of CpGs to gain DNA methylation with time as compared with loss. However, this is likely due to the bias of the $450 \mathrm{k}$ array towards CpG-rich regions. After all, in a previous study comparing wholegenome bisulphite sequencing DNA methylation data of newborn versus a centenarian observed a much higher fraction of CpGs to be hypomethylated in the centenarian than hypermethylated [25].

A limitation of our study is that not all datasets were equally sized. Larger datasets will have higher statistical power to detect aDMPs with smaller effect sizes. However, we showed that there was no relationship between sample size and the tissue-specific character of aDMPs as this was preserved if aDMPs were identified only based on effect size, in equally sized datasets, or in 16 instead of 7 tissues. Also, one would expect at least to find an overlap between the strongest associated aDMPs, but this was not the case. Inspection of effect sizes showed that CpGs detected as aDMPs in one tissue commonly showed little or no evidence for an age-related change in DNA methylation in other tissues. However, larger studies are required to definitely exclude smaller effects in other tissues.

Another limitation of our study is that the age ranges across the different tissues were different, which will have influenced the number of aDMPs detected. Age-related DNA methylation changes are known to accumulate faster during adolescence than in adulthood [38, 39]. This may have contributed the identification of the largest number of aDMPs in the smallest dataset, namely buccal ( $n=96$; age range $1-28)$. Finally, our results may be influenced by measured and unmeasured confounding, such as smoking, BMI, ethnicity and shifts in cell heterogeneity [7]. Some of the changes identified here may also be the result of a shift in cellular composition of a tissue with age, although we adjusted for cellular heterogeneity in brain (neuronal and non-neuronal), monocytes, Th cells (residual impurities) and blood.

\section{Conclusion}

Together, our results show that while the individual CpGs that exhibit age-related differential methylation are highly tissue-specific, the type of functional genomic elements involved are highly consistent across tissues. Gain of methylation occurs at CGIs repressed by PRC2, while loss of methylation accumulates at regions with active histone marks. Our findings indicate that the precision of age predictors based on DNA methylation will depend on whether the tissue of interest was among the tissues on which set the predictor was trained on. Our catalogue of tissue-specific aDMPs may guide the development of more precise predictors of chronological and perhaps eventually provide insight into the tissue-specific differences in the mechanisms underlying ageing.

\section{Methods \\ Datasets}

Datasets used in this study are summarized in Additional file 1: Table S1 and were obtained from the Gene Expression Omnibus or ArrayExpress. For each of the datasets, normalized data or raw IDAT files were obtained. IDAT files of DLPFC samples (age range 0-97 years) were downloaded (GEO accession number: GSE74193). Initial QC was performed using the R package MethylAid [40]. Raw data underwent quality control using a custom pipeline (for more details see https:/git.lumc.nl/ molepi/Leiden450K). Briefly, data were normalized using functional normalization $(\min f i)$, and probes were set to missing if ambiguously mapped, had a high detection $P$ value ( $>0.01)$, low bead count $(<3$ beads) or low success rate (missing in $>95 \%$ of the samples). Normalized data of buccal (GEO accession number: GSE50759, normalization: SWAN method on M values) consisted of 1202 individuals with an age range of 1-28 years. Liver data consisted of 147 individuals with age range between 15 and 86, normalized data of 56 individuals (GEO accession number: GSE48325, normalization using control probes), normalized data of 32 individuals (GEO accession number: GSE61258, normalization using control probes), IDAT files (Level 1) of 30 samples from TCGA and IDAT files of 29 samples were kindly provided by the authors (GSE60753). Given that the liver dataset consisted of dataset from different origins, we carefully inspected for batch effects influencing the age-related changes. The first principal components associated with study, sex and age. To limit the effect of data origin, study ID was added to the model. IDAT files (Level 1) of kidney consisted of 171 individuals with an age range between 15 and 86 and were obtained from TCGA. Normalized data of monocytes (GEO accession number: GSE56046, normalization: quantile normalization per colour signal and probe type) consisted of 1,202 individuals with an age range of 44-83 years. Normalized data of Th cells (GEO accession number: GSE56047, normalization: quantile normalization) consisted of 214 individuals with an age range of 45-79 years). Normalized data of subcutaneous 
fat (Array Expression accession number: E-MTAB-1866, quantile normalized per probe type) consisted of 648 individuals with an age range of 39-85 years. Normalized data of multiple brain regions consisted between 25 and 41 individuals with an age range between 15 and 114 years (GEO accession number: GSE64509, normalization using control probes). Raw IDAT files of epidermis and dermis consisted 38 and 40 individuals between 20 and 90 years (GEO accession number: GSE52980). Normalized data of skeletal muscle consisted of 48 individuals with an age range between 18 and 89 years (GEO accession number: GSE50498, normalization: quantile normalization on $M$ values). Raw IDAT files of thyroid were kindly provided by the authors and consisted of 28 individuals between 23 and 81 years (GEO accession number: GSE53051).

\section{Blood dataset}

DNA methylation blood data consisted of 3,295 from six Dutch biobanks, previously described [23]. Briefly, data were normalized using functional normalization ( $R$ package minfi) using five principal components [41], poorly performing and ambiguously mapped CpGs were removed as well as CpGs on the sex chromosomes. Combat was used to remove residual batch effects [42].

\section{Gene expression}

Gene counts were obtained from GTEX for frontal cortex (for brain-aDMPs measured in DLPFC), oesophagus mucosa (for buccal-aDMPs), liver, kidney cortex and whole blood (for Th cell-aDMPs and monocyte-aDMPs). The package $c q n$ was used to normalize for GC content and gene length. Normalized data was used to calculate the average RPKM per tissue and gene.

\section{Statistical analysis}

aDMPs were identified using linear regression between DNA methylation and age, with adjustment for covariates (sex, gender (all but SAT, females only), dataset (liver), tissue cell composition (DLPFC, monocytes, Th cells, whole blood). For monocytes and Th cells, residual cell impurities were included in the model. For whole blood, blood cell fractions were included as previously described [23]. Age was included in the model as a non-transformed numeric variable. aDMPs were used in subsequent analyses if the slope was higher than $2 \%$ gain or loss per 10 years and if the Bonferroni adjusted $P$ value reached significance $\left(P_{\text {bonf }} \leq 0.05\right)$. To investigate the relation between power and the observed tissue-specific character of aDMPs, we identified aDMPs solely based on the effect size (age-related slope $>2 \% / 10$ year, no $P$ value cut-off). Secondly, a random set of individuals was drawn from each of the tissue datasets with the size equal to the smallest dataset $(N=96)$. aDMPs were identified on these equally sized datasets with both an effect size ( $>2 \% / 10$ years) and $P$ value criterion $\left(P_{\text {bonf }} \leq 0.05\right)$.

\section{Annotations}

CpGs were mapped to $\mathrm{CpG}$ islands (UCSC), shores (2-kb regions flanking regions) and non-CGI described previously [43]. Chromatin state segmentations were obtained from the Epigenomics Roadmap. For each tissue studied, the same tissue or the closest analogue was used from the Roadmap data. For the DLPFC, E073/ DLPFC was used; buccal, E058/keratinocyte foreskin; liver, E066/liver; kidney, E086/foetal kidney; SAT, E063/ Adipose nuclei; monocytes, E029/Monocytes; Th cells, E043/Th cells. For blood, functionality of a certain regions was based on the most frequent occurring feature in primary blood cell subtypes. ChIP-seq data of EZH2 was obtained for all cell types from the ENCODE project. Enrichments were expressed as odds ratio, and $P$ value were calculated using a Chi-squared test. GO enrichment was performed using the default settings of DAVID using nearest genes (UCSC, $3^{\prime}$ or $5^{\prime}$ end of genes closest to the $\mathrm{CpG}$ ) of aDMPs [44].

\section{Additional files}

Additional file 1: Table S1. Number of individuals used per tissue in this study.

Additional file 2: Table S2. Identified aDMPs per tissue.

Additional file 3: Figure S1. A Number of aDMPs ( $y$ axis) in our study against the previously reported number of stem cell divisions per year ( $x$ axis) [47]. B Number of aDMPs ( $y$ axis) against the sample size ( $x$ axis).

Additional file 4: Table S3. Number of tissues a gene near gain-aDMPs was found.

Additional file 5: Figure S2. A Heatmap of slopes of aDMPs identified with only an effect size criterion. $\mathbf{B}$ Heatmap of slopes of aDMPs identified in equally sized datasets comprising randomly selected 96 individuals. Scale represents the change in DNA methylation in \%/10 years. $C$ Number of significant $(P<0.001)$ aDMPs in the other tissues in the equally-sized datasets.

Additional file 6: Figure S3. Heatmap of slopes of age-related DNA methylation in 16 tissues. Scale represents the change in DNA methylation in \%/10 years.

Additional file 7: Figure S4. Overlap between gain- and loss-aDMPs and the CpGs in Horvath's clock.

Additional file 8: Figure S5. Chronological age against the Horvath's predicted age for each of the 16 tissues.

Additional file 9: Figure S6. Volcano plots of all CpGs per tissue, agerelated change ( $x$ axis) versus $P$ value (y axis). $C p G$ from Horvath's age predictor are marked in blue.

Additional file 10: Figure S8. A Percentage (top) and odds ratios (bottom) of gain-aDMPs in CGIs, shores and non-CGls. Blue enriched, red depleted, grey non-significant. $\mathbf{B}$ Percentage (top) and odds ratios (bottom) of aDMPs in EZH2 binding sites in the seven tissues plus whole blood (ChIP-seq, any cell type, ENCODE). Blue enriched, red depleted, grey 
non-significant. C Enrichment of gain-aDMPs in chromatin segmentations expressed in the seven tissues plus whole blood as an odds ratio, grey non-significant. D Percentage (top) and odds ratios (bottom) of loss-aDMPs in CGIs, shores and non-CGls. Blue enriched, red depleted, grey non-significant. E Enrichment of loss-aDMPs in chromatin segmentations expressed in the seven tissues plus whole blood as an odds ratio, grey non-significant. Abbreviations: TssA, Active TSS; TssAFInk, Flanking active TSS; TxFInk, Transcr. at gene 5' and 3'; Tx, Strong transcription; TxWk, Weak transcription; EnhG, Genic enhancers; Enh, Enhancers; ZNF/Rpts, ZNF genes + repeats; Het, Heterochromatin; TssBiv, Bivalent/Poised TSS; BivFlnk, Flanking bivalent TSS/Enh; EnhBiv, Bivalent enhancer; ReprPC, Repressed Polycomb; ReprPCWk, Weak repressed Polycomb, Quies, Quiescent/low.

Additional file 11: Figure S7. A Percentage (top) and enrichment (odds ratio, bottom) for $\mathrm{CGl}$ and the polycomb protein $\mathrm{EZH} 2$ and the combination. $\mathbf{B}$ Expression (y axis, RPKM) of genes near gain- and loss-aDMPs for each tissue for each age category ( $x$ axis).

Additional file 12: Table S4. Frequency of gain-aDMPs near genes. Additional file 13: Table S5. Enriched GO terms for gain-aDMPs per tissue.

Additional file 14: Table S6. Number of tissues a gene near loss-aDMPs was found.

Additional file 15: Table S7. Frequency of loss-aDMPs near genes. Additional file 16: Table S8. Enriched GO terms for loss-aDMPs per tissue.

\section{Abbreviations}

aDMPs: age-related differentially methylated positions; BivFlnk: flanking bivalent transcription start site or enhancer; DLPFC: dorsolateral prefrontal cortex; Enh: enhancers; EnhBiv: bivalent enhancer; EnhG: genic enhancers; Het: heterochromatin; SAT: subcutaneous fat; Th cells: Thelper cells; PcG: polycomb group; PRC2: polycomb repressive complex 2; Quies: quiescent/ low; ReprPC: repressed polycomb; ReprPCWk: weak repressed polycomb; TssA: active TSS; TssAFInk: flanking active TSS; TssBiv: bivalent/poised transcription start site; Tx: strong transcription; TxFlnk: transcribed at gene $5^{\prime}$ and $3^{\prime} ;$ TxWk: weak transcription; ZNF/Rpts: zinc fingers genes and repeats.

\section{Authors' contributions}

RCS, BTH designed the study. RCS peformed the analyses. RCS, BTH, CR, TG contributed to methodology. RCS, BTH contributed to investigation. RCS, BTH wrote the original draft. RCS, BTH, CR, TG, PES helped in writing review and editing. All authors read and approved the final manuscript.

\section{Author details}

${ }^{1}$ Molecular Epidemiology, Department of Biomedical Data Sciences, Leiden University Medical Center, Einthovenweg 20, 2333 ZC Leiden, The Netherlands. ${ }^{2}$ MRC Integrative Epidemiology Unit, School of Social and Community Medicine, University of Bristol, Bristol BS8 2BN, UK.

\section{Acknowledgements}

Not applicable.

\section{Competing interests}

The authors declare that they have no competing interests.

\section{Availability of data and materials}

All datasets used are publically available in GEO under accession numbers E-MTAB-1866, GSE48325, GSE50498, GSE50759, GSE52980, GSE53051, SE55763, GSE56047, GSE56047, GSE60753, GSE61258, GSE64509, GSE74193, GTEX (www.gtexportal.org/home), and TCGA (https://cancergenome.nih.gov).

\section{Consent for publication}

Not applicable.

\section{Ethics approval and consent to participate}

All patients provided consent as described in the respective articles.

\section{Funding}

This study has been funded by the European Union's Seventh Framework Program IDEAL (FP7/2007-2011) under Grant Agreement No. 259679 and the BBSRC (BBI025751/1 and BB/I025263/1). The MRC Integrative Epidemiology Unit receives funding from the UK Medical Research Council (MC_ UU_12013/2, MC_UU_12013/8).

\section{Publisher's Note}

Springer Nature remains neutral with regard to jurisdictional claims in published maps and institutional affiliations.

Received: 21 December 2017 Accepted: 21 May 2018

Published online: 30 May 2018

\section{References}

1. Johansson Å, Enroth S, Gyllensten U. Continuous aging of the human DNA methylome throughout the human lifespan. PLOS ONE. 2013:8:e67378.

2. McClay JL, Aberg KA, Clark SL, Nerella S, Kumar G, Xie LY, Hudson AD, Harada A, Hultman CM, Magnusson PK. A methylome-wide study of aging using massively parallel sequencing of the methyl-CpG-enriched genomic fraction from blood in over 700 subjects. Hum Mol Genet. 2014;23:1175-85

3. Steegenga WT, Boekschoten MV, Lute C, Hooiveld GJ, de Groot PJ, Morris TJ, Teschendorff AE, Butcher LM, Beck S, Müller M. Genome-wide agerelated changes in DNA methylation and gene expression in human PBMCs. Age. 2014;36:1523-40.

4. Garagnani P, Bacalini MG, Pirazzini C, Gori D, Giuliani C, Mari D, Di Blasio AM, Gentilini D, Vitale G, Collino S. Methylation of ELOVL2 gene as a new epigenetic marker of age. Aging Cell. 2012;11:1132-4.

5. Florath I, Butterbach K, Müller H, Bewerunge-Hudler M, Brenner H. Crosssectional and longitudinal changes in DNA methylation with age: an epigenome-wide analysis revealing over 60 novel age-associated CpG sites. Hum Mol Genet. 2014;23:1186-201.

6. Marttila S, Kananen L, Häyrynen S, Jylhävä J, Nevalainen T, Hervonen A, Jylhä M, Nykter M, Hurme M. Ageing-associated changes in the human DNA methylome: genomic locations and effects on gene expression. BMC Genom. 2015;16:179

7. Rakyan VK, Down TA, Maslau S, Andrew T, Yang T-P, Beyan H, Whittaker P, McCann OT, Finer S, Valdes AM. Human aging-associated DNA hypermethylation occurs preferentially at bivalent chromatin domains. Genome Res. 2010;20:434-9.

8. Bell JT, Tsai P-C, Yang T-P, Pidsley R, Nisbet J, Glass D, Mangino M, Zhai G, Zhang F, Valdes A. Epigenome-wide scans identify differentially methylated regions for age and age-related phenotypes in a healthy ageing population. PLoS Genet. 2012;8:e1002629.

9. Yuan T, Jiao Y, de Jong S, Ophoff RA, Beck S, Teschendorff AE. An integrative multi-scale analysis of the dynamic DNA methylation landscape in aging. PLoS Genet. 2015;11:e1004996.

10. Dozmorov MG. Polycomb repressive complex 2 epigenomic signature defines age-associated hypermethylation and gene expression changes. Epigenetics. 2015;10(6):484-95.

11. Rönn T, Volkov P, Gillberg L, Kokosar M, Perfilyev A, Jacobsen AL, Jørgensen SW, Brøns C, Jansson P-A, Eriksson K-F. Impact of age, BMI and $\mathrm{HbA1c}$ levels on the genome-wide DNA methylation and mRNA expression patterns in human adipose tissue and identification of epigenetic biomarkers in blood. Hum Mol Genet. 2015:24:3792-813.

12. Hernandez DG, Nalls MA, Gibbs JR, Arepalli S, van der Brug M, Chong S, Moore M, Longo DL, Cookson MR, Traynor BJ. Distinct DNA methylation changes highly correlated with chronological age in the human brain. Hum Mol Genet. 2011;20:1164-72.

13. Fernández AF, Bayón GF, Urdinguio RG, Toraño EG, García MG, Carella A, Petrus-Reurer S, Ferrero C, Martinez-Camblor P, Cubillo I. H3K4me1 marks DNA regions hypomethylated during aging in stem and differentiated cells. Genome Res. 2015;25(1):27-40.

14. Bekaert B, Kamalandua A, Zapico SC, Van de Voorde W, Decorte R. Improved age determination of blood and teeth samples using a selected set of DNA methylation markers. Epigenetics. 2015;10:922-30. 
15. Bacalini MG, Deelen J, Pirazzini C, De Cecco M, Giuliani C, Lanzarini C, Ravaioli F, Marasco E, van Heemst D, Suchiman HED. Systemic ageassociated DNA hypermethylation of ELOVL2 gene: in vivo and in vitro evidences of a cell replication process. J Gerontol A Biol Sci Med Sci. 2017;72(8):1015-23.

16. Gopalan S, Carja O, Fagny M, Patin E, Myrick JW, McEwen LM, Mah SM, Kobor MS, Froment A, Feldman MW. Trends in DNA methylation with age replicate across diverse human populations. Genetics. 2017;206:1659-74.

17. Spiers H, Hannon E, Wells S, Williams B, Fernandes C, Mill J. Age-associated changes in DNA methylation across multiple tissues in an inbred mouse model. Mech Ageing Dev. 2016;154:20-3.

18. Hannum G, Guinney J, Zhao L, Zhang L, Hughes G, Sadda S, Klotzle B, Bibikova M, Fan JB, Gao Y, et al. Genome-wide methylation profiles reveal quantitative views of human aging rates. Mol Cell. 2013;49:359-67.

19. Weidner Cl, Lin Q, Koch CM, Eisele L, Beier F, Ziegler P, Bauerschlag DO, Jöckel K-H, Erbel R, Mühleisen TW. Aging of blood can be tracked by DNA methylation changes at just three CpG sites. Genome Biol. 2014;15:R24.

20. Horvath S. DNA methylation age of human tissues and cell types. Genome Biol. 2013;14:R115.

21. Christensen BC, Houseman EA, Marsit CJ, Zheng S, Wrensch MR, Wiemels $\mathrm{JL}$, Nelson HH, Karagas MR, Padbury JF, Bueno R. Aging and environmental exposures alter tissue-specific DNA methylation dependent upon CpG island context. PLoS Genet. 2009;5:e1000602.

22. Day K, Waite LL, Thalacker-Mercer A, West A, Bamman MM, Brooks JD, Myers RM, Absher D. Differential DNA methylation with age displays both common and dynamic features across human tissues that are influenced by CpG landscape. Genome Biol. 2013;14:R102.

23. Slieker RC, van Iterson M, Luijk R, Beekman M, Zhernakova DV, Moed $\mathrm{MH}$, Mei H, van Galen M, Deelen P, Bonder MJ, et al. Age-related accrual of methylomic variability is linked to fundamental ageing mechanisms. Genome Biol. 2016;17:191.

24. Horvath S. DNA methylation age of human tissues and cell types. Genome Biol. 2013;14:1.

25. Heyn H, Li N, Ferreira HJ, Moran S, Pisano DG, Gomez A, Diez J, SanchezMut JV, Setien F, Carmona FJ. Distinct DNA methylomes of newborns and centenarians. Proc Natl Acad Sci. 2012;109:10522-7.

26. Kundaje A, Meuleman W, Ernst J, Bilenky M, Yen A, Heravi-Moussavi A, Kheradpour P, Zhang Z, Wang J, Ziller MJ. Integrative analysis of 111 reference human epigenomes. Nature. 2015;518:317-30.

27. Peters MJ, Joehanes R, Pilling LC, Schurmann C, Conneely KN, Powell J Reinmaa E, Sutphin GL, Zhernakova A, Schramm K. The transcriptional landscape of age in human peripheral blood. Nat Commun. 2015;6:8570.

28. Teschendorff AE, Menon U, Gentry-Maharaj A, Ramus SJ, Weisenberger DJ, Shen H, Campan M, Noushmehr H, Bell CG, Maxwell AP. Age-dependent DNA methylation of genes that are suppressed in stem cells is a hallmark of cancer. Genome Res. 2010;20:440-6.

29. Maegawa S, Hinkal G, Kim HS, Shen L, Zhang L, Zhang J, Zhang N, Liang S, Donehower LA, Issa J-PJ. Widespread and tissue specific age-related DNA methylation changes in mice. Genome Res. 2010;20:332-40.

30. Jung M, Pfeifer GP. Aging and DNA methylation. BMC Biol. 2015;13:7.

31. Vandiver AR, Irizarry RA, Hansen KD, Garza LA, Runarsson A, Li X, Chien AL, Wang TS, Leung SG, Kang S. Age and sun exposure-related widespread genomic blocks of hypomethylation in nonmalignant skin. Genome Biol. 2015;16:80
32. Zhou W, Dinh HQ, Ramjan Z, Weisenberger DJ, Nicolet CM, Shen H, Laird PW, Berman BP. DNA methylation loss in late-replicating domains is linked to mitotic cell division. Nat Genet. 2018;50(4):591-602.

33. Koch CM, Joussen S, Schellenberg A, Lin Q, Zenke M, Wagner W. Monitoring of cellular senescence by DNA-methylation at specific CpG sites. Aging Cell. 2012;11:366-9.

34. Koch CM, Reck K, Shao K, Lin Q, Joussen S, Ziegler P, Walenda G, Drescher W, Opalka B, May T. Pluripotent stem cells escape from senescenceassociated DNA methylation changes. Genome Res. 2013:23:248-59.

35. Cruickshanks HA, McBryan T, Nelson DM, VanderKraats ND, Shah PP, van Tuyn J, Rai TS, Brock C, Donahue G, Dunican DS. Senescent cells harbour features of the cancer epigenome. Nat Cell Biol. 2013;15:1495-506.

36. Timp W, Feinberg AP. Cancer as a dysregulated epigenome allowing cellular growth advantage at the expense of the host. Nat Rev Cancer. 2013;13:497-510.

37. Irizarry RA, Ladd-Acosta C, Wen B, Wu Z, Montano C, Onyango P, Cui H, Gabo K, Rongione M, Webster M. The human colon cancer methylome shows similar hypo-and hypermethylation at conserved tissue-specific CpG island shores. Nat Genet. 2009;41:178-86.

38. Kananen L, Marttila S, Nevalainen T, Kummola L, Junttila I, Mononen N, Kähönen M, Raitakari OT, Hervonen A, Jylhä M, et al. The trajectory of the blood DNA methylome ageing rate is largely set before adulthood: evidence from two longitudinal studies. AGE. 2016;38:65.

39. Alisch RS, Barwick BG, Chopra P, Myrick LK, Satten GA, Conneely KN, Warren ST. Age-associated DNA methylation in pediatric populations. Genome Res. 2012;22:623-32.

40. van Iterson M, Tobi E, Slieker R, den Hollander W, Luijk R, Slagboom P, Heijmans B. MethylAid: visual and interactive quality control of large Illumina $450 \mathrm{k}$ data sets. Bioinformatics. 2014;30(23):3435-7.

41. Aryee MJ, Jaffe AE, Corrada-Bravo H, Ladd-Acosta C, Feinberg AP, Hansen KD, Irizarry RA. Minfi: a flexible and comprehensive bioconductor package for the analysis of infinium DNA methylation microarrays. Bioinformatics. 2014;30(10):1363-9.

42. Johnson WE, Li C, Rabinovic A. Adjusting batch effects in microarray expression data using empirical Bayes methods. Biostatistics. 2007:8:118-27.

43. Slieker RC, Bos SD, Goeman JJ, Bovée J, Talens RP, van der Breggen R, Suchiman H, Lameijer E-W, Putter H, van den Akker EB. Identification and systematic annotation of tissue-specific differentially methylated regions using the Illumina 450 k array. Epigenet Chromatin. 2013;6:26.

44. Huang DW, Sherman BT, Lempicki RA. Systematic and integrative analysis of large gene lists using DAVID bioinformatics resources. Nat Protoc. 2009;4:44-57.

45. Thompson RF, Atzmon G, Gheorghe C, Liang HQ, Lowes C, Greally JM, Barzilai N. Tissue-specific dysregulation of DNA methylation in aging. Aging Cell. 2010;9:506-18.

46. Stubbs TM, Bonder MJ, Stark A-K, Krueger F, von Meyenn F, Stegle O, Reik W. Multi-tissue DNA methylation age predictor in mouse. Genome Biol. 2017:18:68.

47. Tomasetti C, Vogelstein B. Variation in cancer risk among tissues can be explained by the number of stem cell divisions. Science. 2015;347:78-81.

Ready to submit your research? Choose BMC and benefit from

- fast, convenient online submission

- thorough peer review by experienced researchers in your field

- rapid publication on acceptance

- support for research data, including large and complex data types

- gold Open Access which fosters wider collaboration and increased citations

- maximum visibility for your research: over 100M website views per year

At BMC, research is always in progress.

Learn more biomedcentral.com/submissions 